\title{
Influence of magnetic order on phonon spectra of multiferroic orthorhombic $\mathrm{YMnO}_{3}$
}

\author{
S.A. Nikolaev ${ }^{\text {a,* }}$, V.G. Mazurenko ${ }^{\text {a }}$, A.N. Rudenko ${ }^{\text {b }}$ \\ a Department of Theoretical Physics and Applied Mathematics, Ural Federal University, 19 Mira str., 620002 Ekaterinburg, Russia \\ ${ }^{\mathrm{b}}$ Institute of Chemical Reaction Engineering, Hamburg University of Technology, 38 Eissendorfer str., 21073 Hamburg, Germany
}

\section{A R T I C L E I N F O}

\section{Article history:}

Received 28 March 2013

Accepted 30 March 2013

by A.H. MacDonald

Available online 6 April 2013

Keywords:

A. Ferroelectrics

A. Magnetically ordered materials

D. Phonons

\begin{abstract}
A B S T R A C T
We perform a first-principles study of lattice dynamics in the low-temperature $P 2_{1} n m$ phase of orthorhombic $\mathrm{YMnO}_{3}$. By considering several possible antiferromagnetic types, we show how magnetic ordering of the system affects its vibrational properties. We find that the experimentally observed magnetic E-type corresponds to the most energetically favorable state and yields phonon spectra, which are consistent with experimental observations. The influence of on-site Coulomb correlations on phonon spectra is also examined. Such effects produce noticeable changes in the spectra and provide a step toward a more accurate description of lattice dynamics in $\mathrm{YMnO}_{3}$.
\end{abstract}

(c) 2013 Elsevier Ltd. All rights reserved.

\section{Introduction}

In magnetic crystals, exchange interactions give rise to a wide variety of magnetic configurations, resulting in different features of their phonon spectra. Earlier studies have reported a frequency shift of the long-wave optic phonons in ferromagnetic insulators, caused by magnetic alignment [1], as well as the change of vibrational frequencies, intensities and half-widths of IR spectra triggered by the change in magnetic order [2]. The question of what features in phonon spectra are attributed to magnetic interactions has recently become more actual due to the great interest in studies of multiferroics.

Multiferroic materials, where magnetism and ferroelectricity coexist, have attracted increasing attention because of a wide variety of magnetic and ferroelectric phase transitions, occurring upon changing temperature and magnetic field. Moreover, spinlattice coupling specifies their unusual properties for spintronic materials. In particular, orthorhombic rare-earth manganites represent an important class of improper multiferroics where the electronic polarization is induced by magnetic order $[3,4]$.

Orthorhombic $\mathrm{YMnO}_{3}$ exhibits two phase transitions: the first one to the antiferromagnetic (AFM) E-type configuration below the Nèel temperature $T_{N}=42 \mathrm{~K}$ and the second one to the ferroelectric phase below the Curie temperature $T_{C}=30 \mathrm{~K}$ [5]. In the temperature range $T_{C}<T<T_{N}$, magnetic moments located on $\mathrm{Mn}^{+3}$ ions form an incommensurate structure with a temperature-independent

\footnotetext{
${ }^{*}$ Corresponding author. Tel.: +7912 6592160

E-mail address: saishi@inbox.ru (S.A. Nikolaev).
}

modulation vector. Below the Curie temperature, the AFM E-type ordering along the $b$-axes becomes more energetically favorable. The phase transition from the paraelectric Pnma structure to the ferroelectric $P 2_{1} \mathrm{~nm}$ one results in the breaking of inversion symmetry and a rise of the macroscopic polarization along the $a$-axes $[5,6]$.

To study these phase transitions and determine their microscopic mechanisms, the temperature dependencies of structural parameters, magnetization, IR and Raman spectra and other properties of orthorhombic $\mathrm{YMnO}_{3}$ have been measured $[5,6]$. Among different features, the appearance of a spectral peak of $620 \mathrm{~cm}^{-1}$ at temperatures less than $T_{F E}$ has been observed. Furthermore, several studies have presented the results of experimental measurements of IR and Raman spectra at room temperatures [7-10], as well as the calculations of dispersive curves and phonon densities of states with the use of empirical methods for the description of interatomic forces [10,11]. However, the existing calculations of the lattice dynamics have been carried out only for the high-temperature Pnma structure. A reliable theoretical description of the low-temperature phase is more challenging due to the increasing role of cooperative effects, such as, for example, phonon-magnon coupling. These effects represent great interest and can be treated by employing first-principles methods only. Moreover, first principles methods allow to treat properly the effects of strong electron correlations, which are known to play an important role in multiferroics [4].

This work is devoted to the first-principles investigation of lattice dynamics and related properties for different magnetic types of the orthorhombic ferroelectric $P 2_{1} \mathrm{~nm}$ phase of $\mathrm{YMnO}_{3}$. The rest of the paper is organized as follows. In Section 2 we describe computational details of our work. Section 3 is devoted to 
the analysis and discussions on the data obtained. Finally, a brief summary of the work is given in Section 4.

\section{Computational details}

In our calculations, we adopt the experimental structure obtained by a single-crystal synchrotron X-ray diffraction [5] as a starting point. At the first step, we consider four types of AFM configurations: the AFM-A with FM (AFM) intraplanar (interplanar) coupling; the AFM-C with FM (AFM) interplanar (intraplanar) coupling; the AFM-G with AFM in both intra- and interplanar couplings and the AFM-E, which represent a combination of antiferromagnetically coupled in-plane FM zig-zag chains with AFM interplanar coupling (Fig. 1). The first three types contain 20 per unit cell, whereas the last one has 40 atoms per unit cell. For lattice dynamics calculations, we consider only E- and A-types, since the first one corresponds to the experimentally observed and theoretically predicted magnetic ordering in orthorhombic $\mathrm{YMnO}_{3}$, while the last one has been subjected to previous studies. Moreover, the A-type is a groundstate magnetic configuration in orthorhombic manganites $\mathrm{RMnO}_{3}$ with light rare-earth cations $(\mathrm{R}=\mathrm{La}, \mathrm{Pr}$ and $\mathrm{Nd}$ ) [4]. As one can see below, these two types are more energetically favorable in comparison with the other types listed above.

Calculations of the lattice dynamics and all the related properties of $\mathrm{YMnO}_{3}$ presented in this work are based on density functional theory. The exchange-correlation functional of the generalized gradient approximation [12] together with the PAW formalism [13] as implemented in the Vienna ab initio simulation package (VASP) [14] have been employed in all calculations. Construction of the dynamical matrices has been performed within the "frozen phonon" framework as implemented in the Phonopy package [15]. To take into account the on-site Coulomb correlations between $d$-electrons of $\mathrm{Mn}^{+3}$ ions and to investigate their effects on phonon spectra, the GGA+U scheme [16] with the values of $U_{\text {eff }}=U-J=0,2$ and $6 \mathrm{eV}$ has been used. For each magnetic ordering, both cell shape and atomic positions have been optimized with $\Gamma$-centered $k$-point meshes of $4 \times 5 \times 3$ and $4 \times 5 \times 5$, respectively for the E-type and the other magnetic configurations. The plane wave cutoff has been set to $500 \mathrm{eV}$, the convergence criteria for the total energy and force calculations to $10^{-10} \mathrm{eV}$ and $10^{-9} \mathrm{eV}$, respectively. To calculate real space force constants, we used $1 \times 2 \times 1$ supercells with 40 atoms per cell in all cases under consideration.

Since the $P 2{ }_{1} \mathrm{~nm}$ structure of $\mathrm{YMnO}_{3}$ is polar, it is important to properly treat a non-analytical contribution of the dynamical matrix that results in LO-TO splitting. The non-analytical part
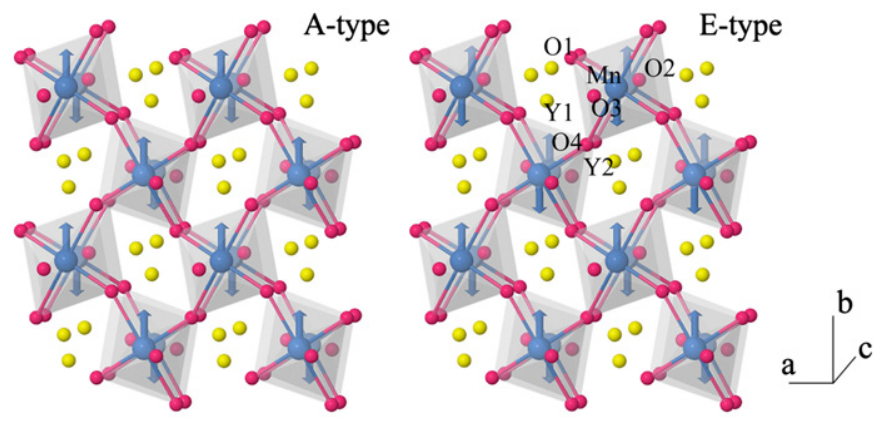

Fig. 1. (Color online) Low-temperature $P 2{ }_{1} \mathrm{~nm}$ structure with the AFM A- and E-types. Blue, red and yellow spheres denote manganese, oxygen and yttrium respectively. has the general form

${ }^{\mathrm{NA}} D_{i j}^{\mu \nu}(\mathbf{q} \rightarrow 0)=D_{i j}^{\mu \nu}(\mathbf{q} \rightarrow 0)+\frac{4 \pi}{\sqrt{M_{\mu} M_{\nu}} \Omega_{0}} \frac{\left(\sum_{k} q_{k} Z_{\mu, k i}^{*}\right)\left(\sum_{k^{\prime}} q_{k^{\prime}} Z_{\nu, k^{\prime} j}^{*}\right)}{\sum_{i j} q_{i} \epsilon_{i j}^{\infty} q_{j}}$,

where $M_{\mu}$ is the mass of the $\mu$-ion, $\Omega_{0}$ is the volume of the unit cell, $Z_{\mu, i j}^{*}$ is the Born effective charge defined as the microscopic polarization with respect to a structural displacement

$Z_{\mu, i j}^{*}=\Omega_{0} \frac{\partial P_{i}}{\partial e_{j}^{\mu}(\mathbf{q}=0)}$,

and $\epsilon_{i j}^{\infty}$ is the electronic dielectric-constant tensor, which is defined as the derivative of the polarization with respect to a microscopic electric field

$\epsilon_{i j}^{\infty}=\delta_{i j}+\left.4 \pi \frac{\partial P_{i}}{\partial E_{j}}\right|_{e^{\mu}(q=0)=0}$.

The calculation of the Born effective charges and the dielectric tensors has been performed within the linear-response method of density-functional perturbation theory [17].

\section{Results and discussion}

Total energy calculations yield the AFM E-type ordering to be the most energetically favorable phase among all magnetic types listed above (Table 1). However, on-site Coulomb interaction applied to $\mathrm{Mn} d$-electrons stabilizes the AFM A-type, when $U$ parameter is greater than $2 \mathrm{eV}$. This result is in line with previous reports [6]. Magnetic texture of the E-type breaks inversion symmetry even for the centrosymmetric Pnma phase, so the relaxed structure retains its ionic positions according to the noncentrosymmetric $P 2_{1} \mathrm{~nm}$ group. On the contrary, for the A-type, which does not break inversion symmetry, structural optimization leads to the centrosymmetric structure. As a result, all the corresponding bond lengths and angles in $\mathrm{MnO}_{6}$ octahedra become equal for the A-type. The Born effective charge tensors and high-frequency static dielectric tensors for the AFM A- and E-types calculated with $U=0 \mathrm{eV}$ are presented in Table 2. The data obtained differ considerably from their nominal ionic values, which, in turn, results in the presence of large dynamical contributions to the static charge because of atomic displacements of the ferroelectric phase transition. Inversion symmetry break occurring during the phase transition leads to anisotropic Born charge's diagonal components; this fact alongside with nonzero non-diagonal components suggests strong bonding in $\mathrm{MnO}_{6}$ octahedra. The presence of anomalously large Born charges due to strong oxygen-transition metal bonding is one of the main features of ferroelectric perovskites [18].

Calculated values of the Born charges are quite similar for two magnetic types considered, but several differences can be mentioned. As one can see, values obtained for the A-type are almost equal for pairs $\mathrm{Y} 1-\mathrm{Y} 2, \mathrm{O} 1-\mathrm{O} 4$ and $\mathrm{O} 2-03$. As has been previously mentioned, this is due to structural optimization, after which the relaxed structure nearly coincides in Wyckoff notations with the high-temperature Pnma phase. In contrast, the Born charges of the E-type are unequal for each ion because of distortions of $\mathrm{MnO}_{6}$ octahedron from its symmetric form. Then, on-site Coulomb

Table 1

Calculated energies (in $\mathrm{eV} /$ formula unit) for different antiferromagnetic types of orthorhombic $\mathrm{YMnO}_{3}$ and on-site Coulomb parameter $U$.

\begin{tabular}{lllll}
\hline$U(\mathrm{ev})$ & E-type & A-type & C-type & G-type \\
\hline 0 & $\mathbf{- 4 3 . 5 5 5}$ & -43.541 & -43.529 & -43.538 \\
2 & $\mathbf{- 4 2 . 5 1 6}$ & -42.511 & -42.498 & -42.496 \\
6 & -40.772 & $\mathbf{- 4 0 . 7 9 4}$ & -40.760 & -40.748 \\
\hline
\end{tabular}


interaction of $d$-electrons enhances their localization and slightly decreases the Born charge's values. This fact has an impact on calculations of macroscopic polarization with the $G G A+U$, where the on-site repulsion of $d$-electrons decreases electronic polarization. As to the dielectric tensor, its change within the GGA+U scheme is more noticeable, about $20-30 \%$ with respect to $U=0 \mathrm{eV}$.

Based on the force constants and non-analytical corrections, phonon densities of states and dispersion curves for the AFM A- and E-types are calculated. In the vicinity of the $\Gamma$ point acoustic vibrational modes become slightly imaginary, that is hardly seen in the scale of Fig. 2. This is related to limitations of the computational method, known for the low-frequency range. The use of larger supercells can solve this problem, but it will lead to much more expensive computations. Therefore the effect of the imaginary modes is supposed to be negligible and we can exclude them from our considerations. However, it is worth noting that the presence of soft modes in ferroelectrics is driven by their intrinsic instability, where anharmonicity plays a fundamental role, and calculations in the harmonic approximation at zero temperature may result in imaginary values [17]. We can assume that an exact description of the low-frequency range in the vibrational spectra needs a more rigorous approach such as density functional perturbation theory. Nevertheless, one can see that the phonon spectrum of the A-type exhibits some modes with soft behavior along the X-S, X-R and Z-R lines, which may indicate intrinsic instability in the system and allows us to conclude that the E-type is more dynamically stable phase. In addition, it should be emphasized that there is a difference in the LO-TO splitting, which is more pronounced for the AFM A-type, where all three directions, $\Gamma-\mathrm{X}, \Gamma-\mathrm{Y}$ and $\Gamma$-Z, exhibit different behaviors.

Phonon densities of states (Fig. 3) calculated without nonanalytical corrections taken into account show a shift toward high frequencies in passing from the A- to E-type due to the increase of bond lengths in $\mathrm{MnO}_{6}$ octahedra, which affects interatomic force constants. Given that the elements of the dynamical matrices are proportional to squares of the corresponding frequencies, vibrational shifts of the E-type can be evaluated as about 3-5\% with respect to the A-type. By comparing phonon densities calculated with different $U$ values, one can also see a slight shift of the vibrational spectra toward the low-frequency range. The shift related to on-site Coulomb correlations can be associated with the decreasing oxygen-manganese coupling in the $\mathrm{MnO}_{6}$ octahedron. From Fig. 3 one can see the importance of non-analytical corrections coming from the long-range dipole-dipole interaction. Their contributions significantly change the final shape of phonon spectra, particularly for the A-type. Finally, as follows from the partial phonon densities of states (Fig. 4), vibrations of yttrium and

Table 2

Calculated Born effective charge tensors and high-frequency static dielectric tensors for the AFM A- and E-types with $U=0$ eV.

\begin{tabular}{|c|c|c|c|c|c|c|c|c|c|}
\hline & $x x$ & $x y$ & $x z$ & $y x$ & $y y$ & $y z$ & $z x$ & $z y$ & $z z$ \\
\hline \multicolumn{10}{|c|}{ E-type } \\
\hline Y1 & 3.716 & 0.001 & 0.002 & 0.0 & 3.743 & -0.294 & 0.0 & 0.785 & 4.282 \\
\hline Y2 & 3.746 & 0.0 & -0.002 & 0.0 & 3.638 & -0.077 & 0.0 & 0.675 & 3.941 \\
\hline Mn & 3.771 & -0.426 & -0.375 & -0.441 & 3.079 & -0.616 & 0.243 & -0.160 & 3.763 \\
\hline 01 & -1.994 & -0.059 & -0.038 & 0.072 & -2.037 & 0.344 & -0.179 & -0.042 & -2.639 \\
\hline $\mathrm{O} 2$ & -3.460 & 0.004 & -0.004 & 0.0 & -1.717 & 0.366 & 0.0 & 0.018 & -2.111 \\
\hline $\mathrm{O} 3$ & -3.552 & 0.003 & -0.003 & 0.0 & -1.743 & 0.009 & 0.0 & 0.193 & -1.903 \\
\hline $\mathrm{O} 4$ & -1.999 & -0.017 & -0.099 & -0.097 & -3.013 & 1.099 & -0.090 & 0.814 & -3.235 \\
\hline$\epsilon^{\infty}$ & 7.123 & 0.0 & 0.0 & 0.0 & 9.075 & 0.0 & 0.0 & 0.0 & 8.720 \\
\hline \multicolumn{10}{|c|}{ A-type } \\
\hline Y1 & 3.703 & 0.001 & 0.0 & 0.0 & 3.614 & -0.297 & 0.0 & 0.832 & 4.196 \\
\hline Y2 & 3.720 & 0.001 & 0.0 & 0.0 & 3.620 & -0.298 & 0.0 & 0.834 & 4.194 \\
\hline Mn & 3.796 & -0.342 & -0.358 & -0.869 & 2.719 & -0.527 & 0.321 & -0.063 & 3.977 \\
\hline 01 & -2.011 & -0.081 & -0.070 & 0.189 & -2.282 & 0.948 & -0.360 & 0.153 & -3.106 \\
\hline $\mathrm{O} 2$ & -3.494 & -0.002 & 0.002 & 0.0 & -1.773 & 0.034 & 0.0 & 0.083 & -1.958 \\
\hline $\mathrm{O} 3$ & -3.489 & -0.002 & 0.002 & 0.0 & -1.770 & 0.037 & 0.0 & 0.081 & -1.958 \\
\hline 04 & -2.007 & -0.080 & -0.069 & 0.191 & -2.285 & 0.954 & -0.359 & 0.153 & -3.106 \\
\hline$\epsilon^{\infty}$ & 7.256 & 0.0 & 0.0 & 0.0 & 10.254 & 0.0 & 0.0 & 0.0 & 10.010 \\
\hline
\end{tabular}

Table 3

Wyckoff notations and irreducible representations (also their correspondence with the Pnma group (No. 62)) for the low-temperature $P 2_{1} \mathrm{~nm}$ (No. 31) structure of $\mathrm{YMnO}_{3}$.

\begin{tabular}{lll}
\hline Atom & Wyckoff symbol & Irreducible representations \\
\hline $\mathrm{Y} 1$ & $2 \mathrm{a}$ & $2 \mathrm{~A}_{1} \oplus \mathrm{A}_{2} \oplus \mathrm{B}_{1} \oplus 2 \mathrm{~B}_{2}$ \\
$\mathrm{Y} 2$ & $2 \mathrm{a}$ & $2 \mathrm{~A}_{1} \oplus \mathrm{A}_{2} \oplus \mathrm{B}_{1} \oplus 2 \mathrm{~B}_{2}$ \\
$\mathrm{Mn}$ & $4 \mathrm{~b}$ & $3 \mathrm{~A}_{1} \oplus 3 \mathrm{~A}_{2} \oplus 3 \mathrm{~B}_{1} \oplus 3 \mathrm{~B}_{2}$ \\
$\mathrm{O} 1$ & $4 \mathrm{~b}$ & $3 \mathrm{~A}_{1} \oplus 3 \mathrm{~A}_{2} \oplus 3 \mathrm{~B}_{1} \oplus 3 \mathrm{~B}_{2}$ \\
$\mathrm{O} 2$ & $2 \mathrm{a}$ & $2 \mathrm{~A}_{1} \oplus \mathrm{A}_{2} \oplus \mathrm{B}_{1} \oplus 2 \mathrm{~B}_{2}$ \\
$\mathrm{O} 3$ & $2 \mathrm{a}$ & $2 \mathrm{~A}_{1} \oplus \mathrm{A}_{2} \oplus \mathrm{B}_{1} \oplus 2 \mathrm{~B}_{2}$ \\
$\mathrm{O} 4$ & $4 \mathrm{~b}$ & $3 \mathrm{~A}_{1} \oplus 3 \mathrm{~A}_{2} \oplus 3 \mathrm{~B}_{1} \oplus 3 \mathrm{~B}_{2}$ \\
& & \\
$\mathrm{~A}_{1} \longrightarrow \mathrm{A}_{g} \mathrm{~B}_{1 u}$ & & $\Gamma=17 \mathrm{~A}_{1} \oplus 13 \mathrm{~A}_{2} \oplus 13 \mathrm{~B}_{1} \oplus 17 \mathrm{~B}_{2}$ \\
$\mathrm{~A}_{2} \longrightarrow \mathrm{A}_{u} \mathrm{~B}_{1 g}$ & & $\Gamma_{\mathrm{RAMAN}}=16 \mathrm{~A}_{1} \oplus 13 \mathrm{~A}_{2} \oplus 12 \mathrm{~B}_{1} \oplus 16 \mathrm{~B}_{2}$ \\
$\mathrm{~B}_{1} \longrightarrow \mathrm{B}_{3 g} \mathrm{~B}_{2 u}$ & & $\Gamma_{\mathrm{IR}}=16 \mathrm{~A}_{1} \oplus 13 \mathrm{~B}_{1} \oplus 16 \mathrm{~B}_{2}$ \\
$\mathrm{~B}_{2} \longrightarrow \mathrm{B}_{2 g} \mathrm{~B}_{3 u}$ & & $\Gamma_{\mathrm{ACOUSTI}}=\mathrm{A}_{1} \oplus \mathrm{B}_{1} \oplus \mathrm{B}_{2}$ \\
\hline
\end{tabular}




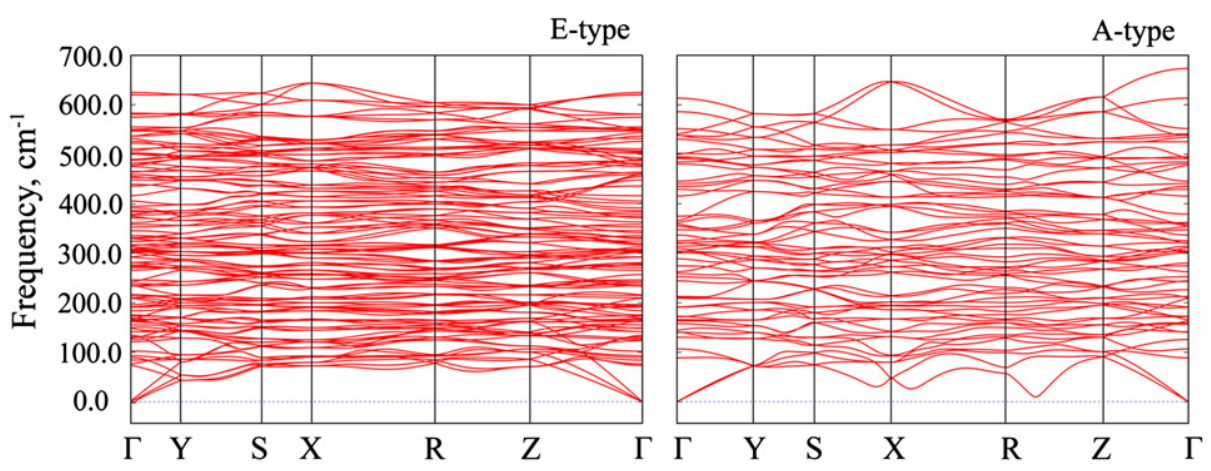

Fig. 2. (Color online) Calculated dispersion curves for the AFM E- (left) and A- (right) types with $U=0 \mathrm{eV}$.
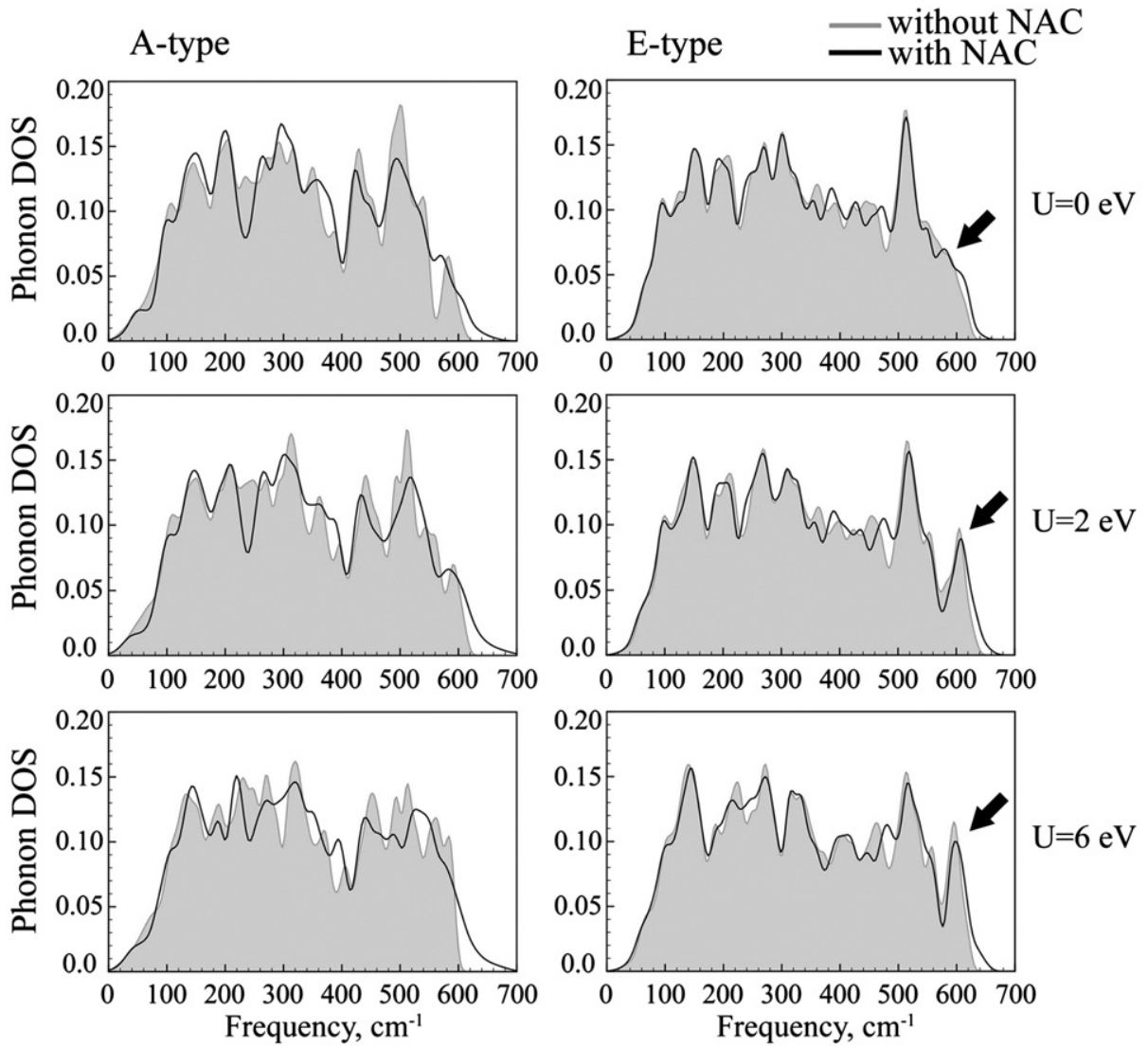

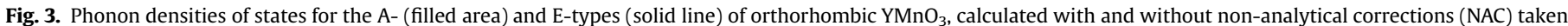
into account. Black arrows indicate the presence of high-frequency peak.

manganese ions lie in the low-frequency range $\left(0-400 \mathrm{~cm}^{-1}\right)$, whereas the main contribution in the high-frequency range comes from oxygen vibrations $\left(200-600 \mathrm{~cm}^{-1}\right)$.

One of the most general features of the calculated phonon spectra is the presence of two peaks $\left(520 \mathrm{~cm}^{-1}\right.$ and $\left.610 \mathrm{~cm}^{-1}\right)$ in the high-frequency range for the AFM E-type ordering (Fig. 4), with the main contribution coming from vibrations of $\mathrm{MnO}_{6}$ octahedra. In particular, the contribution from $\mathrm{O} 2$ and $\mathrm{O} 3$ ions is dominant for both peaks. Magnetic order in the system plays a substantial role in the origin of these peaks. By considering several magnetic types, it can be seen that the high-frequency peak at $610 \mathrm{~cm}^{-1}$ (II in Fig. 4) appears only for the experimentally observed E-type configuration. The peak at $520 \mathrm{~cm}^{-1}$ (I in Fig.4) appears for both magnetic configurations, but more pronounced in the E-type case also. Consideration of the on-site Coulomb repulsion makes the second high-frequency peak more pronounced for $U \geq 2 \mathrm{eV}$ and his value of $610 \mathrm{~cm}^{-1}$ obtained for $U=2 \mathrm{eV}$ is in a good agreement with the experimentally reported peak of $620 \mathrm{~cm}^{-1}$ [19]. This indicates an important role of strong electron correlations for the lattice dynamics of orthorhombic $\mathrm{YMnO}_{3}$. The peak I is also consistent with the experimental observations at $495 \mathrm{~cm}^{-1}$ [19]. In Fig. 5 we present the projected phonon densities of states for oxygen ions calculated with $U=2 \mathrm{eV}$. One can see that the first peak is formed primarily by vibrations of $02-03$ ions along the $a$ axis and 01-04 ions along the $b$ - and $c$-axes, while the peak II arises from $a b$-vibrations of all oxygen ions. As an example, in Fig. 6 we show eigenvectors of the dynamical matrix for the $\Gamma$-point, corresponding to the atomic displacements, associated with the high-frequency peaks. From Fig. 6 it is seen that the peak I corresponds to out-of-phase oxygen vibrations, representing the so-called bending character (with irreducible representation $A_{1}$, $A_{g}(3)$ in [19] Table 3) and the second one is related to in-phase vibrations of oxygen ions in the $a b$ plane, forming a stretching mode (with irreducible representation $B_{2}, B_{2 g}(1)$ in [19]). Finally, it is 

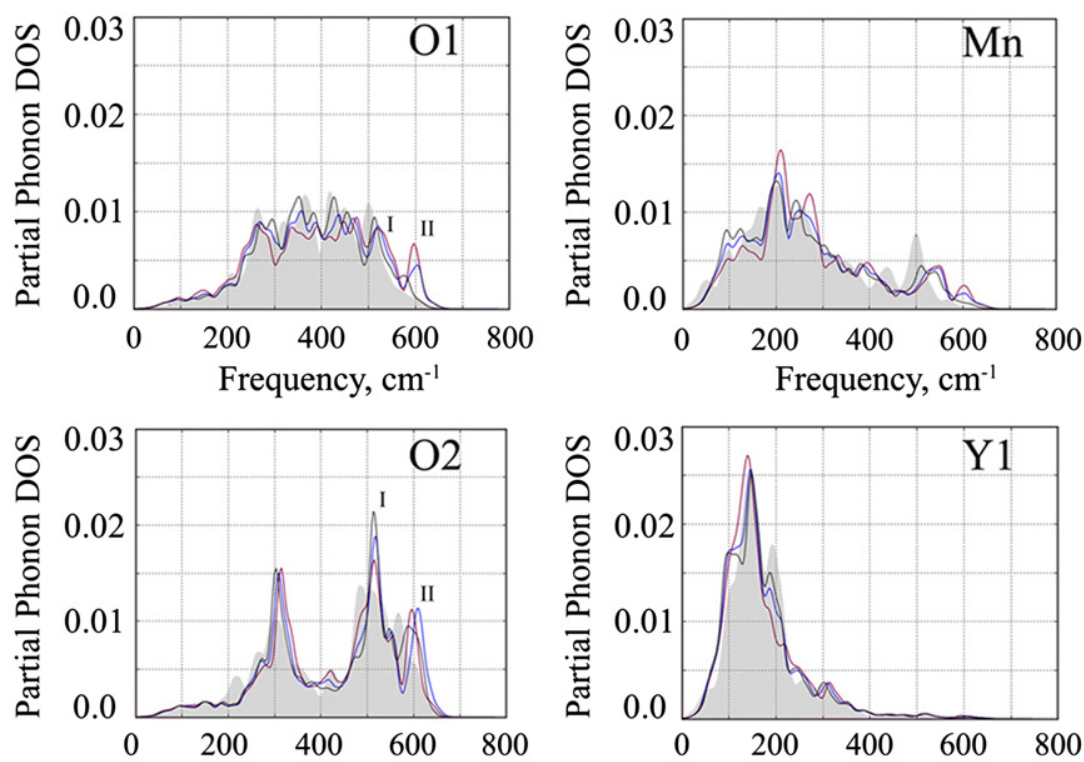

Frequency, $\mathrm{cm}^{-1}$

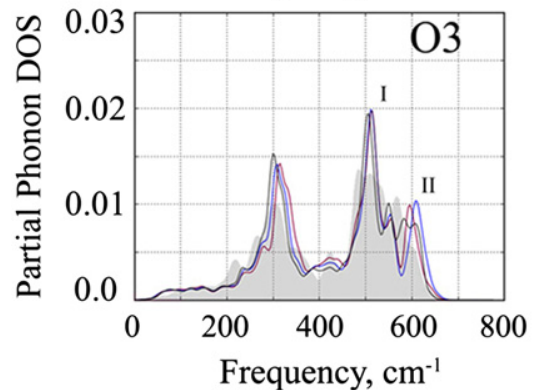

Frequency, $\mathrm{cm}^{-1}$
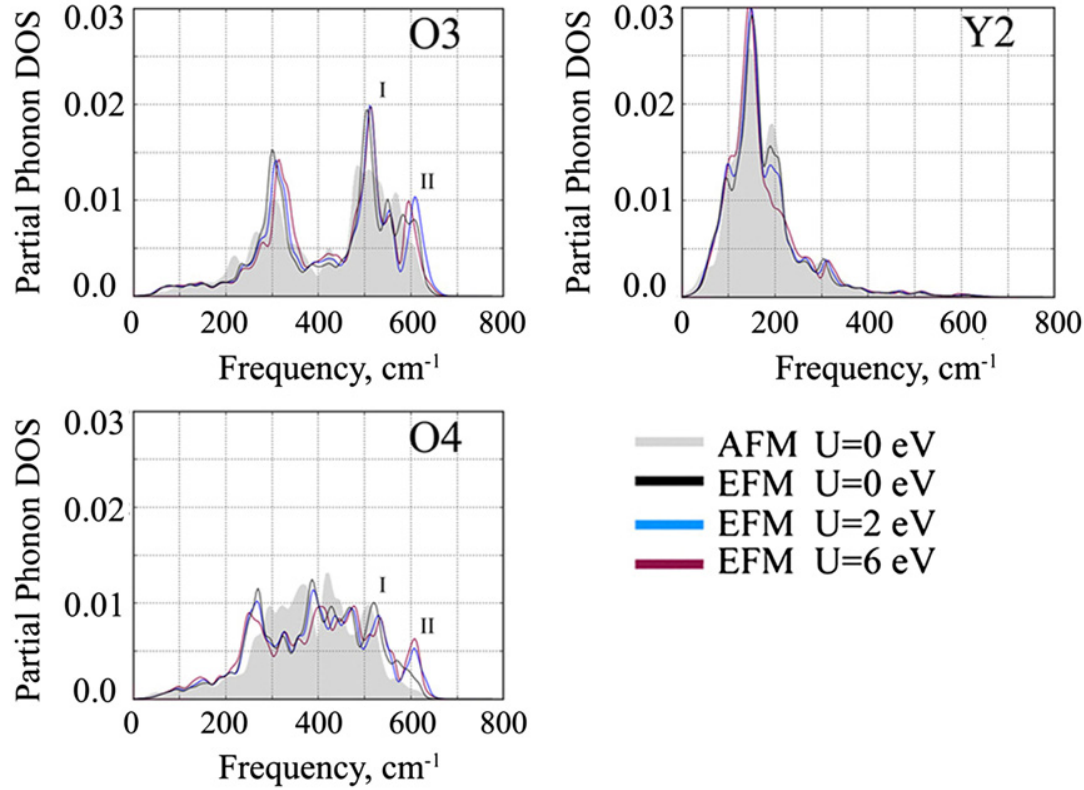

Fig. 4. (Color online) Partial phonon densities of states calculated for the AFM A- (grey area) and E-types (colored lines). I and II denote two high-frequency peaks.
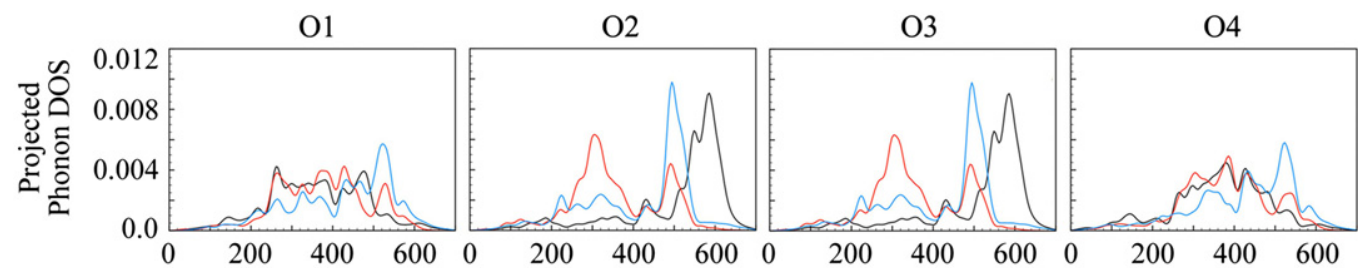

A-type
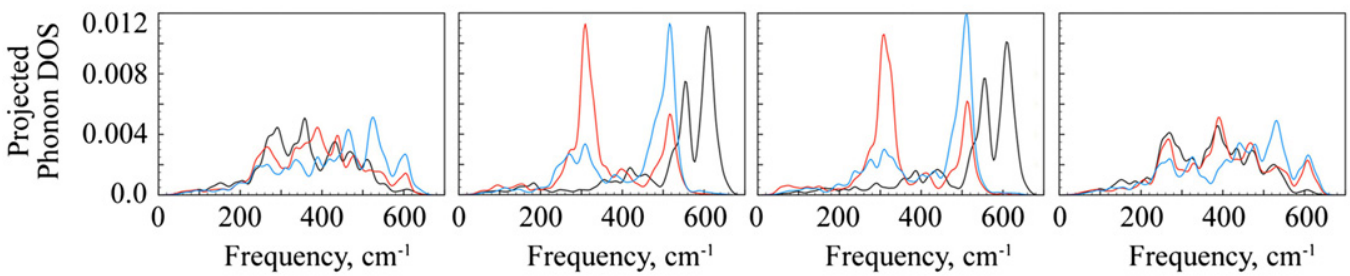

$-\mathrm{X}$
$-\mathrm{Y}$
$-\mathrm{Z}$

E-type

Fig. 5. (Color online) Projected phonon densities of states for oxygen ions in the AFM A- (grey area) and E-types (colored lines) with $U=2 \mathrm{eV}$. 

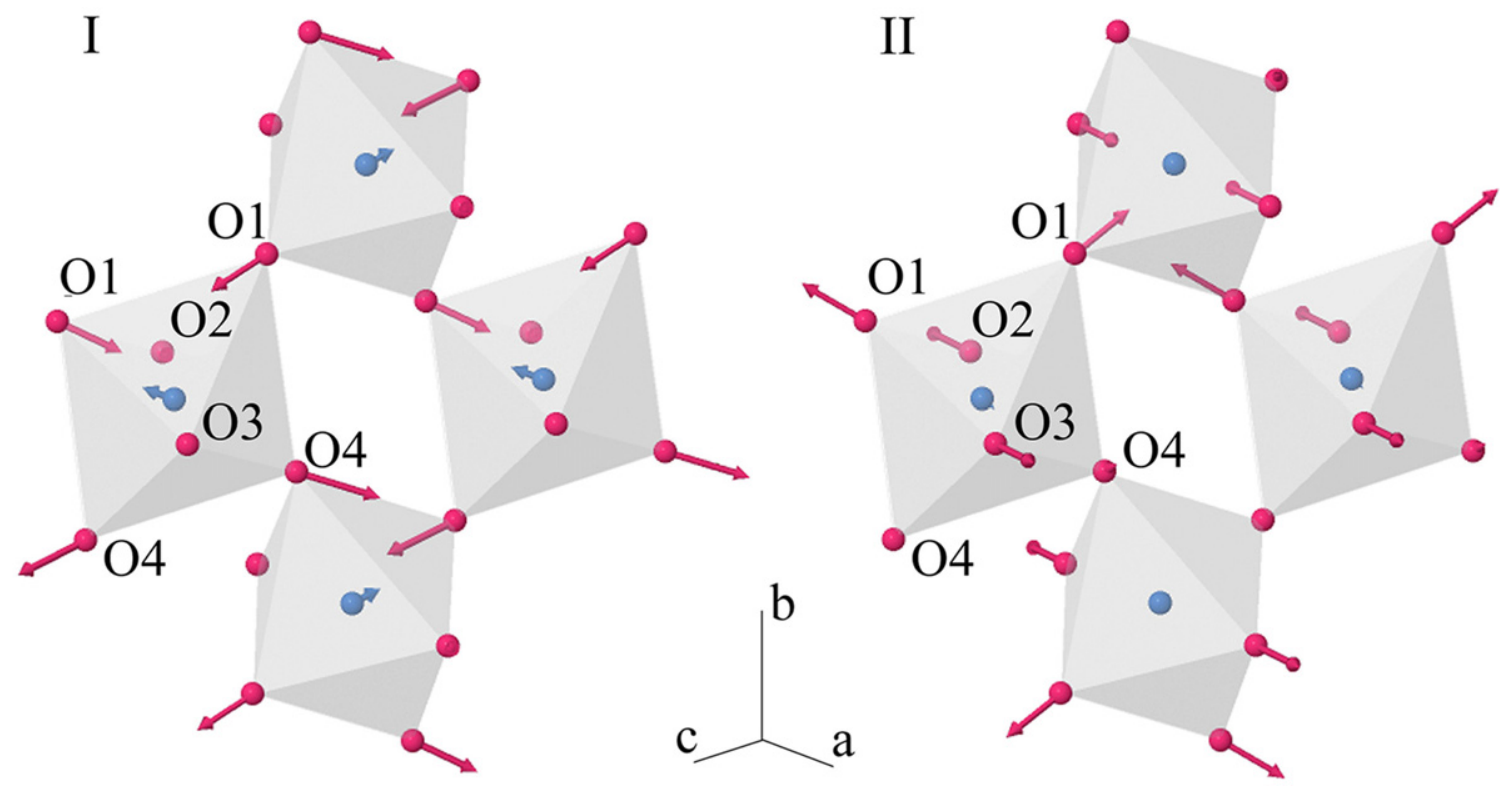

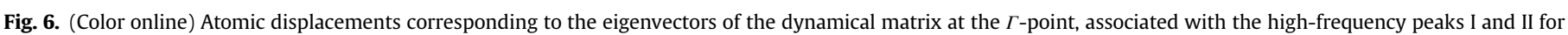
the E-type with $U=2 \mathrm{eV}$.

reasonable to assume that the formation of the peaks is eventually related to the difference in the interatomic bonding between the Eand A-type orderings, associated with magnetic interactions, which in the case of the E-type ordering leads to increasing of bond lengths in $\mathrm{MnO}_{6}$ octahedra and the crystal structure with broken inversion symmetry, as opposed to the A-type.

\section{Summary and conclusions}

We have performed first-principles calculations of lattice dynamics for different antiferromagnetic types in the low-temperature ferroelectric phase of orthorhombic $\mathrm{YMnO}_{3}$.The Born effective charges, high-frequency dielectric tensors and Coulomb parameter $U$ have been taken into account. In accordance with previous reports the collinear antiferromagnetic E-type has been found to be the most energetically stable. Our results show that the phonon spectra are sensitive to magnetic ordering in the system. We have theoretically reproduced the presence of the experimentally observed phonon peak at $620 \mathrm{~cm}^{-1}$, formation of which is associated with the correct E-type magnetic alignment and the ferroelectric phase transition. Finally, we have demonstrated the influence of the on-site Coulomb parameter $U$ and nonanalytical corrections on phonon spectra and their important contributions to a quantitative description of vibrational properties in orthorhombic $\mathrm{YMnO}_{3}$.

It should be noted that, to better understand the connection between magnetic ordering and phonon spectra, investigations covering lattice dynamics in a wider temperature range are required. We hope that our work will stimulate further studies on this important issue.

\section{Acknowledgment}

The work was supported by the Project 14.A18.21.0076 of the Ministry of Education and Science of the Russian Federation.

\section{References}

[1] W. Baltensperger, J.S. Helman, Helv. Phys. Acta 41 (1968) 668.

[2] R. Haumont, J. Kreisel, P. Bouvier, F. Hippert, Phys. Rev. B 73 (2006) 132101.

[3] B. Lorenz, Y.-Q. Wang, C.-W. Chu, Phys. Rev. B 76 (2007) 104405.

[4] K. Yamauchi, F. Freimuth, S. Blugel, S. Picozzi, Phys. Rev. B 78 (2008) 014403.

[5] D. Okuyama, S. Ishiwata, Y. Takahashi, K. Yamauchi, S. Picozzi, K. Sugimoto, H. Sakao, M. Takata, R. Shimano, Y. Taguchi, T. Arima, Y. Tokura, Phys. Rev. B 84 (2011) 054440.

[6] S. Picozzi, K. Yamauchi, G. Bihlmayer, S. Blugel, Phys. Rev. B 74 (2006) 094402.

[7] H.C. Gupta, P. Ashdhir, Physica B 262 (1999) 1.

[8] M.N. Iliev, M.V. Abrashev, Journal of Raman Spectroscopy 21 (2001) 805

[9] J. Laverdièlre, S. Jandl, A.A. Mukhin, V.Y. Ivanov, Eur. Phys. J. B 54 (2006) 67.

[10] M.N. Iliev, M.V. Abrashev, H.-G. Lee, V.N. Popov, Y.Y. Sun, C. Thomsen, R. L. Meng, C.W. Chu, Phys. Rev. B 57 (1998) 2872.

[11] M.N. Rao, N. Kaur, S.L. Chaplot, N.K. Gaur, R.K. Singh, J. Phys.: Condens. Matter 21 (2009) 355402.

[12] J.P. Perdew, K. Burke, M. Ernzerhof, Phys. Rev. Lett. 77 (1996) 3865

[13] P.E. Blochl, Phys. Rev. B 50 (1994) 17953.

[14] G. Kresse, J. Hafner, Phys. Rev. B 47 (1993) 558

[15] A. Togo, F. Oba, I. Tanaka, Phys. Rev. B 78 (2008) 134106.

[16] V. Anisimov, A. Lichtenstein, F. Aryasetiawan, J. Phys.: Condens. Matter 9 (1997) 767.

[17] S. Baroni, S. de Gironcoli, A. Dal Corso, P. Giannozzi, Rev. Mod. Phys. 73 (2001) 515.

[18] M. Posternak, R. Resta, A. Baldereschi, Phys. Rev. B 50 (1994) 8911.

[19] J. Kim, S. Jung, M.S. Park, S.-I. Lee, H.D. Drew, H. Cheong, K.H. Kim, E.J. Choi, Phys. Rev. B 74 (2006) 052406. 